\title{
Effect of internal gravitational coupling on Titan's non-synchronous rotation
}

\author{
Ö. Karatekin, ${ }^{1}$ T. Van Hoolst, ${ }^{1}$ and T. Tokano ${ }^{2}$ \\ Received 20 May 2008; revised 8 July 2008; accepted 16 July 2008; published 27 August 2008.
}

[1] Variations in the spin period of Titan have been detected by the Cassini radar observations. Angular momentum exchange between Titan's surface and the atmosphere over seasonal time scales corresponding to Saturn's orbital period of 29.5 year is the most likely cause of the observed non-synchronous rotation. The measured rotation rate can be explained if Titan's icy crust is rotationally decoupled from the interior in the presence of a subsurface ocean. However, we show that Titan is likely to rotate almost as a rigid body even when it has an internal ocean because of a substantial internal gravitational coupling between the crust and the interior. The predicted surface rotation rate will be further reduced due to Saturn's torque, but can approach to the observed value if other factors, such as larger atmospheric torque, smaller equatorial flattening or viscous relaxation of the icy crust are considered. Citation: Karatekin, Ö, T. Van Hoolst, and T. Tokano (2008), Effect of internal gravitational coupling on Titan's non-synchronous rotation, Geophys. Res. Lett., 35, L16202, doi:10.1029/2008GL034744.

\section{Introduction}

[2] The rotation rate of Saturn's moon Titan is not constant as revealed by Cassini radar observations over several years [Lorenz et al., 2008]. The difference between the present-day rotation period from synchronous spin results in a shift of $0.36^{\circ}$ per year in apparent longitude. This observation is consistent with the seasonal exchange of angular momentum between the surface and Titan's dense superrotating atmosphere and reveals an internal water ocean like that on Europa, if it can be assumed that the rotation of the crust is decoupled from the interior. In reality, the interior and the crust are never completely decoupled. Although the friction forces on the boundaries of the internal ocean can be neglected [Lorenz et al., 2008; Tokano and Neubauer, 2005], the gravitational interaction between the crust and the interior is likely to be large enough to prohibit the relative motion of the crust and interior as shown to be the case for the librations of Europa [Van Hoolst et al., 2008].

[3] Here, we investigate the influence of internal gravitational coupling on the variations in the spin rotation of Titan in response to the atmospheric forcing. In Section 2, analytical solutions for the rotations of the crust and the interior separated by a subsurface ocean are derived which

\footnotetext{
${ }^{1}$ Royal Observatory of Belgium, Brussels, Belgium.

${ }^{2}$ Institut für Geophysik und Meteorologie, Universität zu Köln, Cologne, Germany.
}

Copyright 2008 by the American Geophysical Union. 0094-8276/08/2008GL034744 take into account the internal gravitation coupling. The strength of the gravitational coupling and the amplitude of the non-synchronous surface rotation are calculated for a broad set of interior structure models of Titan. The results are discussed in the light of Cassini radar observations in Section 3.

\section{Surface Rotation With Internal Gravitational Coupling}

[4] The gravitational torque acting against the differential rotation of the crust and the solid interior is a function of their flattening. Titan has an ellipsoidal figure: rotation flattens Titan at the poles creating an equatorial bulge, whereas the tides stretch Titan in the direction to Saturn causing a contraction in the direction perpendicular to Saturn. The equatorial flattening is defined as $\beta(r)=(a(r)$ $-b(r)) / a(r)$ where $a$ and $b$ are the two equatorial radii and $r$ is the mean radius of an ellipsoidal surface. The strength of the gravitational torque for an interior composed of three layers is given by [see Van Hoolst et al., 2008]

$$
K=\frac{4 \pi G}{5} \frac{8 \pi}{15} \rho_{i} \beta_{i} r_{i}^{5}\left[\rho_{c} \beta_{c}+\left(\rho_{o}-\rho_{c}\right) \beta_{o}\right]\left(1-\frac{\rho_{o}}{\rho_{i}}\right),
$$

where $G$ is the universal gravitational constant, $\rho$ the density, and the subscripts $c, o$ and $i$ correspond to the crust, ocean and the interior, respectively. The gravitational torque vanishes when the internal flattening is zero $\left(\beta_{i} \approx 0\right)$.

[5] By using the conservation of angular momentum, the angular motions of the crust and the solid interior can be described by two coupled ordinary differential equations of second order:

$$
\begin{gathered}
C_{c} \ddot{\theta}_{c}=-K \sin 2\left(\theta_{c}-\theta_{i}\right)+\Gamma(t), \\
C_{i} \ddot{\theta}_{i}=K \sin 2\left(\theta_{c}-\theta_{i}\right) .
\end{gathered}
$$

[6] Here $C$ is the polar moment of inertia, $\Gamma(t)$ the timevariable atmospheric torque acting on the surface of Titan and $\theta$ the rotation angle in a reference frame rotating with the mean synchronous rotation. Due to the finite obliquity and eccentricity of the Saturn-Titan system orbiting the Sun, there are variations in the insolation pattern which cause substantial seasonal variation in the zonal wind speed and direction near the surface [Tokano and Neubauer, 2005]. The resulting seasonal variation of the atmospheric torque can be calculated from the exchange of angular momentum between the surface and atmosphere predicted by means of a 3-dimensional general circulation model [Tokano and Neubauer, 2005] as $\Gamma(t)=-d(A A M) / d t$, where $A A M$ is the atmospheric angular momentum. The torque is princi- 

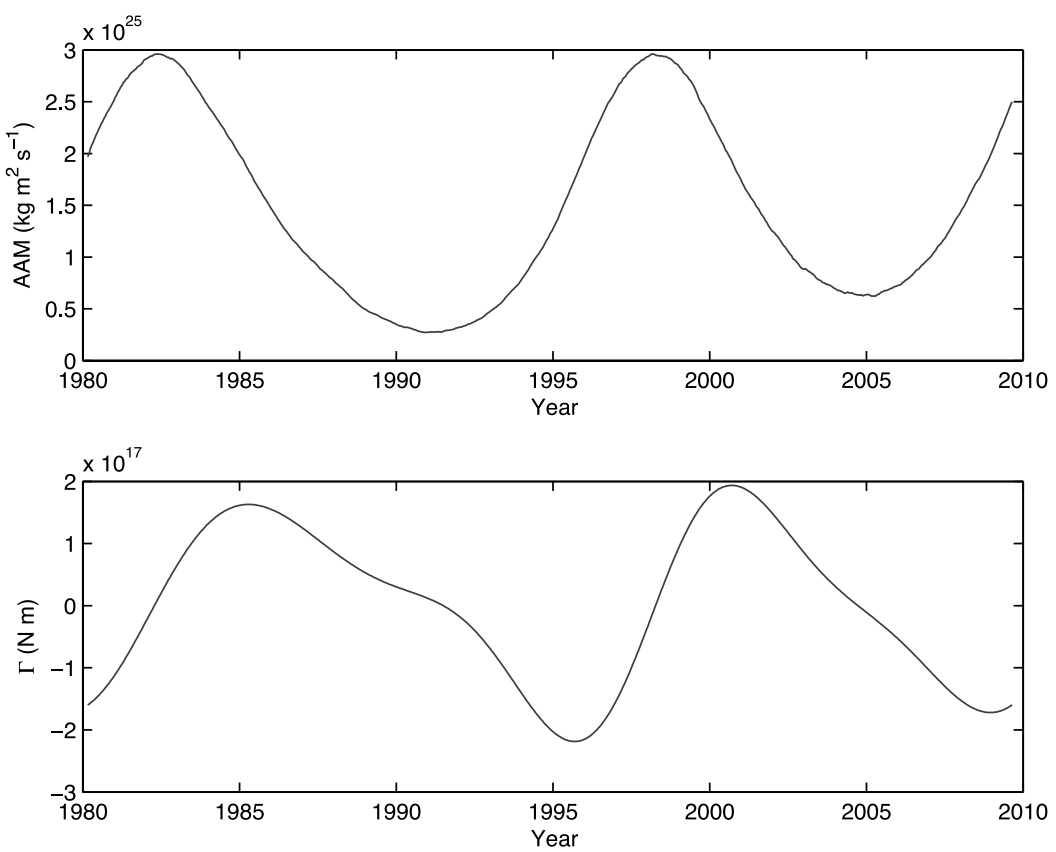

Figure 1. Seasonal variations of the atmospheric angular momentum $A A M$ and torque $\Gamma$ (mean value is removed) calculated from the output of the Titan GCM [Tokano and Neubauer, 2005] as a function of time with origin at the vernal equinox of Saturn in February 1980.

pally semi-annual $\left(\omega_{0}=2 \pi / 14.74\right.$ years $\left.=1.35 \times 10^{-8} \mathrm{~s}^{-1}\right)$ with an amplitude of $\Gamma_{0}=1.6 \times 10^{17} \mathrm{~N}$ m (see Figure 1). The semi-annual components of the $A A M$ and $\Gamma$ are also dominant on the Earth [de Viron et al., 1999] and Mars [Sanchez et al., 2003].

[7] Equations (2) and (3) can be solved at the dominant semi-annual forcing frequency $\left(\Gamma(t)=\Gamma_{0} e^{i \omega_{0}} t\right)$ by assuming small angular differences, so that $\sin 2\left(\theta_{c}-\theta_{i}\right) \approx 2\left(\theta_{c}-\theta_{i}\right)$. We have:

$$
\begin{aligned}
& \theta_{c}=\frac{\Gamma_{0}\left(2 K-\omega_{0}^{2} C_{i}\right)}{\omega_{0}^{2} C_{i}\left(C_{c} \omega_{0}^{2}-2 K \frac{C_{i}+C_{c}}{C_{i}}\right)} e^{i \omega_{0} t}, \\
& \theta_{i}=\frac{2 K \Gamma_{0}}{\omega_{0}^{2} C_{i}\left(C_{c} \omega_{0}^{2}-2 K \frac{C_{i}+C_{c}}{C_{i}}\right)} e^{i \omega_{0} t} .
\end{aligned}
$$

The rotations of both the crust and the interior are amplified resonantly when $2 K\left(C_{i}+C_{c}\right) / C_{i} \approx C_{c} \omega_{0}^{2}$.

[8] Limit cases in which the outer shell rotates either independently or together with the solid interior can be found depending on the relative amplitude of the gravitational coupling strength $2 K\left(C_{i}+C_{c}\right) / C_{i} \approx 2 K$ (the moment of inertia of the crust $C_{c}$ is generally much smaller than that of the interior $C_{i}$ ) with respect to $C_{c} \omega_{0}^{2}$. When the coupling is weak, $K \ll C_{c} \omega_{0}^{2}$, the interior is at rest $\left(\theta_{i} \approx 0\right)$ with respect to the mean rotation of Titan while the outer shell rotation is linearly proportional to the amplitude of the atmospheric torque $\Gamma_{0}$ and inversely proportional to $C_{c}$ :

$$
\theta_{c} \approx-\frac{\Gamma_{0}}{\omega_{0}^{2} C_{c}} e^{i \omega_{0} t}
$$

[9] If the internal gravitational coupling is strong, $K \gg$ $C_{c} \omega_{0}^{2}$, the crust and the interior move together with an amplitude inversely proportional to the sum of moments of inertia of the crust and interior:

$$
\theta_{c} \approx \theta_{i} \approx-\frac{\Gamma_{0}}{\omega_{0}^{2}\left(C_{c}+C_{i}\right)} e^{i \omega_{0} t}
$$

[10] The strength of the gravitational coupling $K$ on Titan depends on the interior structure, which is presently uncertain. Theoretical models suggest a differentiated body with an internal ocean, a several tens of $\mathrm{km}$ thick outer crust composed of water ice, and an interior containing high pressure ices and silicates [Sohl et al., 1995; Grasset et al., 2000; Sohl et al., 2003; Tobie et al., 2005; Fortes et al., 2007]. In order to calculate the plausible range of gravitational coupling strengths we develop interior models of Titan composed of an icy crust $\left(\rho_{c}=920 \mathrm{~kg} \mathrm{~m}^{-3}\right)$ a subsurface ocean $\left(\rho_{o}=1000 \mathrm{~kg} \mathrm{~m}^{-3}\right)$ and a homogenous core. Additional layering of the interior into a high pressure ice and silicate layers have an effect of only a few percent on the gravitational coupling strength. The density and the radius of the core is calculated in order to satisfy the observed mass $\left(M=1.3456 \times 10^{23} \mathrm{~kg}\right)$ and radius $(R=$ $2575 \mathrm{~km})$ for given moment of inertia $\left(0.29<I / M R^{2}<0.38\right)$ and outer shell thickness (80 km $<h<400 \mathrm{~km})$. By assuming the interior of Titan to be in hydrostatic equilibrium, the internal flattening is calculated using the classical Radau equation for rotationally flattened bodies including also tidal deformation.

[11] The strength of the gravitational coupling $K$ given by equation (1) is of the order of $10^{20} \mathrm{Nm}$ for the range of interior models studied. The variation of $K$ with the moment of inertia factor $\left(I / M R^{2}\right)$ for a crust thickness of $120 \mathrm{~km}$ is presented in Figure 2. The flattenings are more pronounced at larger values of $I / M R^{2}$ leading to an increase of $K$ from 


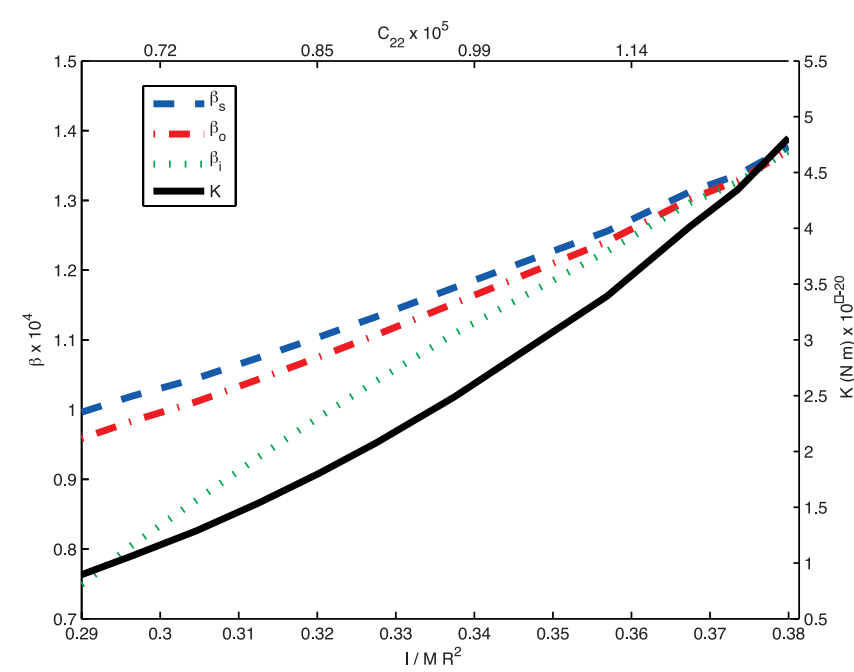

Figure 2. Gravitational coupling strength $K$ and flattening of the surface $\beta_{s}$, bottom of the crust $\beta_{o}$ and bottom of the ocean $\beta_{i}$ as a function of the mean moment of inertia factor $I / M R^{2}$ for a crust thickness of $\mathrm{h}=120 \mathrm{~km}$. The crust and ocean densities are $\rho_{s}=920 \mathrm{~kg} \mathrm{~m}^{-3}$ and $\rho_{o}=1000 \mathrm{~kg} \mathrm{~m}^{-3}$ respectively.

$9.06 \times 10^{19} \mathrm{Nm}$ for $I / M R^{2}=0.29$ to $4.82 \times 10^{20} \mathrm{Nm}$ for $I / M R^{2}=0.38$. In comparison, the gravitational coupling strengths of the interior models proposed by Sohl et al. [1995, 2003] and Fortes et al. [2007] with 4 and 5 layers are $K=1.34 \times 10^{20} \mathrm{Nm}, 1.77 \times 10^{20} \mathrm{Nm}$ and $1.74 \times 10^{20} \mathrm{Nm}$, respectively. Larger crust and ocean densities $\left(\rho_{c}=1063\right.$ $\rho_{o}=1350 \mathrm{kgm}^{-3}$ as suggested by Fortes et al. [2007] decrease the coupling strength presented in Figure 2 from $K \approx 2.6 \times 10^{20} \mathrm{Nm}$ to $K \approx 1.7 \times 10^{20} \mathrm{Nm}$ for $I / M R^{2}=0.34$. The weakest gravitational coupling corresponds to a Titan with low moment of inertia and high density ocean and crust.

[12] As shown above, the response of the crust to atmospheric torque is characterised by the ratio $2 K\left(C_{i}+\right.$ $\left.C_{s}\right) / C_{i} \approx 2 K$ to $\omega_{0}^{2} C_{s}$. The crust is rotationally decoupled from the interior when the ratio is much smaller than unity $\left(K<10^{17} \mathrm{Nm}\right)$ and the rotation variations are resonantly amplified when it is close to unity $\left(K \approx 10^{18} \mathrm{Nm}\right)$. The ratio is between about 10 and 100 for the models studied, suggesting that the gravitational coupling $\left(K \approx 10^{20} \mathrm{Nm}\right)$ is strong enough to lock the rotations of the crust and the solid interior (see equation (7)). For comparison, the ratio is between 50 and 80 for the interior models given in the literature [Sohl et al., 1995, 2003; Fortes et al., 2007].

[13] The departure of Titan's surface rotation from synchronous spin is between $2 \times 10^{-3}<\theta_{c}<6 \times 10^{-3}$ as follows from the analytical solution given by equations (4)-(5) (see Figure 3 ). The largest shift in the apparent longitude is less than $\Delta \lambda<16 \mathrm{~km}$. Figure 4 shows a typical solution with the full time-dependent atmospheric torque $\Gamma(t)$ obtained numerically for $I / M R^{2}=0.36$ and shell thickness of $80 \mathrm{~km}$. This moment of inertia factor is consistent with the preliminary solution of the gravity coefficient $C_{22}=1.12 \times 10^{-5}$ determined from the Doppler tracking of the Cassini spacecraft [Iess et al., 2007] by assuming hydrostatic equilibrium. The rotations of the crust and the interior are seen to be strongly coupled with semi-annual amplitudes of $\left|\theta_{c}\right| \approx$ $3.1 \times 10^{-3} \operatorname{rad}(\Delta \lambda \approx 8 \mathrm{~km})$ and $\left|\theta_{i}\right| \approx 3.3 \times 10^{-3} \mathrm{rad}$ in very good agreement with the analytical solutions (equations (4)-(5)). The small difference between $\left|\theta_{i}\right|$ and $\left|\theta_{c}\right|$ results from the fact that $\omega_{0}^{2} C_{i}$ is not completely negligible with respect to $K$ (see equations (4) and (7)). The corresponding peak rate of rotation for this particular model is $\dot{\theta}_{c} \approx 0.06^{\circ} \mathrm{yr}^{-1}$ in the period 2004-2007 (Figure 4 (bottom)). The semi-annual amplitude of the rotation rate $\left(\dot{\theta}_{c}=\omega_{0} \theta_{c}\right)$ varies between $0.05^{\circ} \mathrm{yr}^{-1}$ and $0.15^{\circ} \mathrm{yr}^{-1}$ for our

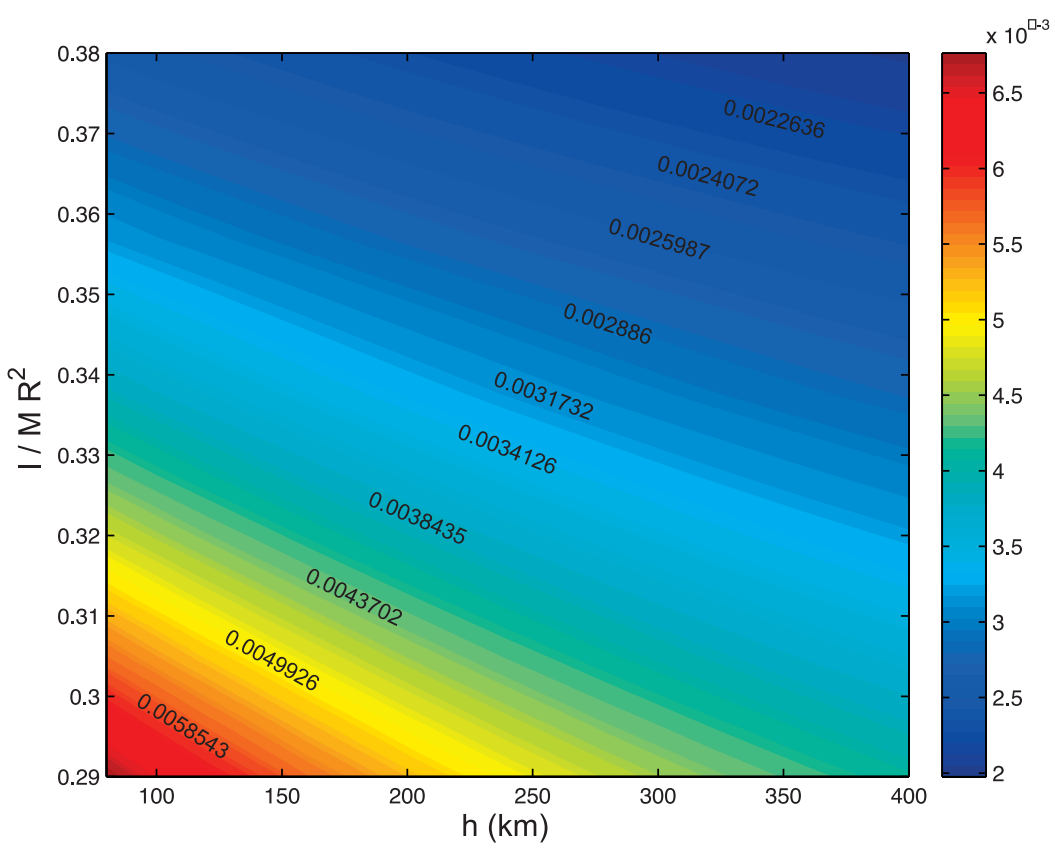

Figure 3. Surface rotation angle $\theta_{c}$ (in radians) given by equation (4) as a function of crust thickness and mean moment of inertia factor for an atmospheric torque $\Gamma(t)=\Gamma_{0} e^{i \omega_{0} t}$ with $\Gamma_{0}=1.6 \times 10^{17} \mathrm{~N} \mathrm{~m}$ and $\omega_{0}=1.35 \times 10^{-8} \mathrm{~s}^{-1}$. 

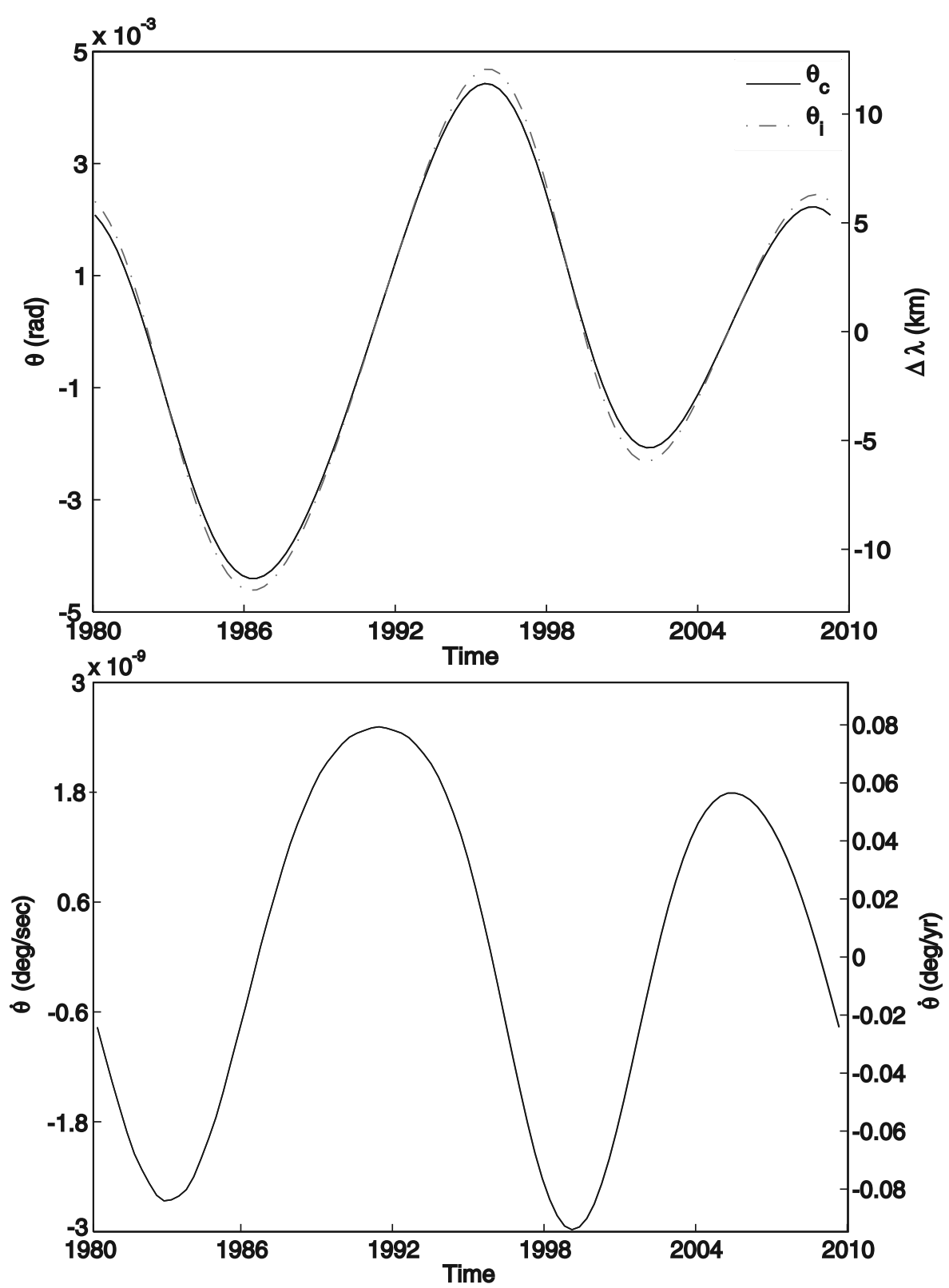

Figure 4. Seasonal changes in (top) the non-synchronous rotation angle of the crust $\theta_{c}$ and (bottom) its time derivative, $\dot{\theta}_{c}$ for $I / M R^{2}=0.36$ and a shell thickness of $80 \mathrm{~km}$. Variation in the non-synchronous rotation of the interior $\theta_{i}$ is plotted in comparison with $\theta_{c}$ in the top plot.

broad set of interior structure models of Titan. The nonsynchronous angular surface displacement rate observed by the Cassini radar between 2004 and 2007 is $\dot{\theta}_{c}=0.36^{\circ} \mathrm{yr}^{-1}$ [Lorenz et al., 2008], between about 2.5 and 7 times larger than predicted here. The smallest differences are obtained for small moment of inertia with a thin crust (see Figure 3).

\section{Discussion}

[14] The interpretation of the observed rate of rotation by neglecting internal couplings led [Lorenz et al., 2008] to conclude that an ocean exists below the surface of Titan. However, we have shown that due to the robust gravitational coupling, the crust and the solid interior are likely to rotate together even with a subsurface ocean. A strong internal coupling would be compatible with the radar measurements of Cassini, if the atmospheric torque were larger by about a factor 2.5 to 7 depending on the interior structure. For small moment of inertia $\left(I / M R^{2} \leq 0.3\right)$ and thin crust $(\leq 150 \mathrm{~km})$, an increase of the atmospheric torque by a factor 2.5 to about $4 \times 10^{17} \mathrm{~N}$ m would be sufficient. For comparison, the atmospheric torque is of the order of $10^{21} \mathrm{~N} \mathrm{~m}$ on the Earth [de Viron et al., 1999], which has smaller surface pressure. The value of the atmospheric torque of Titan determined by Tokano and Neubauer [2005] includes only the effect of the seasonal variations in zonal winds. A more detailed analysis considering other contributions to the atmospheric torque such as from surface pressure variations and the surface topography may enhance the total atmospheric torque. 
[15] Alternatively, with the predicted atmospheric torque by Tokano and Neubauer [2005], the observed rotation rate can be explained if the internal gravitational coupling is at least one order of magnitude smaller $\left(K<10^{19} \mathrm{Nm}\right)$ than the values obtained here. Such a smaller gravitational coupling can be obtained if the flattening of the solid interior would be at least one order of magnitude smaller than the hydrostatic flattening (see Figure 2). Preliminary analysis of the Cassini flybys suggests that Titan's interior is not in hydrostatic equilibrium [Iess et al., 2007], but a departure from hydrostatic equilibrium usually means stronger departure from sphericity and is likely to enhance not only the gravitational coupling but also pressure and friction torques neglected in the present study. Another possibility is that Titan's internal mass distribution does not reflect its present orbital configuration but dates back from earlier times. The difficulty with this scenario is that it requires a larger semimajor axis of Titan by about a factor two.

[16] Other torques can also change the rotation variations of Titan. In particular, Saturn exerts gravitational torques on the a-spherical icy crust, ocean, and interior. Since those torques tend to align the long axes of the internal regions with the direction to Saturn, they reduce the amplitude of the rotation variations induced by the atmosphere. Therefore, the difference between the predicted and observed rotation variations will be several times larger than the differences given above considering internal gravitational coupling only. Other internal coupling mechanisms can either lead to less differential rotation between the internal regions (e.g. due to viscous coupling) or can counteract the gravitational couplings. For example, Mercury's liquid core is thought not to follow the rotation variation, or librations, of the mantle due to the fact that the torque resulting from pressure in the liquid core compensates for the Solar gravitational torque on the core [Van Hoolst, 2007].

[17] The effect of internal and external gravitational torques on the rotation variations could be largely reduced if the viscoelastic icy crust can relax its shape and adjust its orientation to that of the forcing gravitational field. The gravitational torques diminish strongly if the time scale of relaxation of the crust is much smaller than the period of the rotation variations of $14.74 \mathrm{yrs}$. In that case, the rotation variation of the icy crust could be large and close to that of a decoupled crust. Preliminary calculations show that for the lowest possible viscosity, the melting ice viscosity of about $10^{13} \mathrm{~Pa} \mathrm{~s}$, the effective strength of the internal gravitational coupling reduces substantially [Van Hoolst and Karatekin, 2008].

[18] The forced librations in longitude due to the gravitational attraction of Saturn and the finite eccentricity of Titan cause an additional non-synchronous rotation variation with a much shorter period equal to the orbital period of Titan around Saturn (15.9454 day). These librations can also be used to study Titan's interior, however, they may be difficult to detect since the resulting equatorial displacements are much smaller, between about $50 \mathrm{~m}$ for a rigidly rotating Titan [Comstock and Bills, 2003] and several hundred meters for negligible internal coupling compared to shifts of several kilometres due to the atmosphere. Further observations of Titan's rotation over a longer part of the atmospheric cycle with the expected peak in 2009 and the transition to a slower than synchronous rotation in 2013, combined with advances in atmospheric modelling will allow better constraining the nature of the non-synchronous rotation of Titan and the influence of the interior and the atmosphere.

[19] Acknowledgments. O.K. acknowledges the support of the Belgian PRODEX program managed by the European Space Agency in collaboration with the Belgian Federal Science Policy Office. T.T. was supported by DFG.

\section{References}

Comstock, R. L., and B. G. Bills (2003), A solar system survey of forced librations in longitude, J. Geophys. Res., 108(E9), 5100, doi:10.1029/ 2003JE002100.

de Viron, O., C. Bizouard, D. Salstein, and V. Dehant (1999), Atmospheric torque on the Earth and comparison with atmospheric angular momentum variations, J. Geophys. Res., 104(B3), 4861-4875, doi:10.1029/ 1998JB90006.

Fortes, A. D., P. M. Grindrod, S. K. Trickett, and L. Vočadlo (2007), Ammonium sulfate on titan: Possible origin and role in cryovolcanism, Icarus, 188, 139-153, doi:10.1016/j.icarus.2006.11.002.

Grasset, O., C. Sotin, and F. Deschamps (2000), On the internal structure and dynamics of Titan, Planet. Space Sci., 48, 617-636.

Iess, L., J. W. Armstrong, M. di Benedetto, A. Graziani, R. Mackenzie, P. Racioppa, N. Rappaport, and P. Tortora (2007), The determination of Titan's gravity field from Doppler tracking of the Cassini spacecraft, in Proceedings of the 20th International Symposium on Space Flight Dynamics [CD-ROM], NASA Tech. Rep., NASA/CP-2007-214158.

Lorenz, R. D., B. W. Stiles, R. L. Kirk, M. D. Allison, P. P. D. Marmo, L. Iess, J. I. Lunine, S. J. Ostro, and S. Hensley (2008), Titan's rotation reveals an internal ocean and changing zonal winds, Science, 319(5870), 1649-1651, doi:10.1126/science.1151639.

Sanchez, B. V., D. D. Rowlands, R. M. Haberle, and J. Schaeffer (2003), Atmospheric rotational effects on Mars based on the NASA Ames general circulation model, J. Geophys. Res., 108(E5), 5040, doi:10.1029/ 2002JE001984.

Sohl, F., W. Sears, and R. Lorenz (1995), Tidal dissipation on Titan, Icarus, $115,278-294$.

Sohl, F., H. Hussmann, B. Schwentker, T. Spohn, and R. D. Lorenz (2003), Interior structure models and tidal Love numbers of Titan, J. Geophys. Res., 108(E12), 5130, doi:10.1029/2003JE002044.

Tobie, G., O. Grasset, J. Lunine, A. Mocquet, and C. Sotin (2005), Titan's internal structure inferred from a coupled thermal-orbital model, Icarus, 175, 496-502, doi:10.1016/j.icarus.2004.12.007.

Tokano, T., and F. M. Neubauer (2005), Wind-induced seasonal angular momentum exchange at Titan's surface and its influence on Titan's length-of-day, Geophys. Res. Lett., 32, L24203, doi:10.1029/ 2005 GL024456.

Van Hoolst, T. (2007), The rotation of the terrestrial planets, in Treatise on Geophysics, vol. 10, Planets and Moons, edited by T. Spohn, pp. 123164, Elsevier, Amsterdam.

Van Hoolst, T., and Ö. Karatekin (2008), The influence of internal gravitational coupling and ice viscosity on Titan's length-of-day variations, paper presented at European Planetary Science Congress 2008, Inst. for Planetol., Munster, Germany.

Van Hoolst, T., N. Rambaux, Ö. Karatekin, V. Dehant, and A. Rivoldini (2008), The librations, shape, and icy shell of Europa, Icarus, 195, 386399, doi:10.1016/j.icarus.2007.12.011.

Ö. Karatekin and T. Van Hoolst, Royal Observatory of Belgium, 3 Av. Circulaire, B-1180, Brussels, Belgium. (o.karatekin@oma.be)

T. Tokano, Institut für Geophysik und Meteorologie, Universität zu Köln, D-50937 Köln, Germany. 